\title{
Impact of Peri-Operative Anemia and Blood Transfusions in Patients with Gastric Cancer Receiving Gastrectomy
}

\author{
Chih-Chun Chang ${ }^{1 \&}$, Jen-Tang Sun ${ }^{2 \&}$, Jing-Yuan Chen ${ }^{1 \&}$, Yi-Ting Chen ${ }^{1}$, Pei-Yu \\ $\mathbf{L i}^{1}$, Tai-Chen Lee1, Ming-Jang Su${ }^{1}$, Jiann-Ming $\mathrm{Wu}^{3}$, Tzung-Hai Yen ${ }^{4}$, Fang-Yeh \\ $\mathrm{Chu}^{1,5,6 *}$
}

\begin{abstract}
Background: Potential disadvantages of blood transfusion during curative gastrectomy for gastric cancer have been reported, and the role of peri-operative transfusions remains to be ascertained. Thus, the aim of our study was to survey its impact in patients with gastric cancer undergoinging gastrectomy. Materials and Methods: Clinical data of patients receiving curative gastrectomy at Far Eastern Memorial Hospital were obtained. Findings for pre-operative anemia states, pre-, peri- and post-operative transfusion of red blood cell (RBC) products as well as post-operative complication events were collected for univariate analysis. Results: A total of 116 patients with gastric cancer received gastrectomy at Far Eastern Memorial Hospital from 2011 to 2014. Both pre-operative and intra- and post-operative transfusion of RBC products were markedly associated with post-operative infectious events (OR: 3.70,95\% CI: 1.43-9.58, $P=0.002$; OR: $8.20,95 \%$ CI: 3.11-22.62, $P<0.001$, respectively). In addition, peri- and post-operative $\mathrm{RBC}$ transfusion was significantly associated with prolonged hospital stay from admission to discharge (OR: 8.66, 95\% CI: 1.73-83.00, $P=0.002)$ and post-operative acute renal failure (OR: 19.69, 95\% CI: $2.66-854.56, P<0.001)$. Also, the overall survival was seemingly decreased by peri-operative $\mathrm{RBC}$ transfusion in our gastric cancer cases $(\mathrm{P}=\mathbf{0 . 0 7 8})$. Conclusions: Our survey indicated that peri-operative RBC transfusion could increase the risk of infectious events and acute renal failure post curative gastrectomy as well as worsen the overall survival in gastric cancer cases. Hence, unnecessary blood transfusion before, during and after curative gastrectomy should be avoided in patients with gastric cancer.
\end{abstract}

Keywords: Peri-operative transfusion - gastric cancer - gastrectomy - red blood cells

Asian Pac J Cancer Prev, 17 (3), 1427-1431

\section{Introduction}

It was known that tumor resection was often inevitably accompanied with a vast amount of blood loss and required subsequent blood transfusion therapy. Besides, cancer-related anemia commonly occurred in patients with malignancies in the gastrointestinal tract (Ye et al., 2015). Therefore, blood transfusion therapy was frequently required for the correction of low hemoglobin $(\mathrm{Hb})$ level as pre-operative anemia was well known to be associated with poor outcomes of the patients (Tsai et al., 2015).

However, it has been reported that blood transfusion in patients with cancer could be detrimental (Gantt et al., 1981). Recent studies also indicated that allogeneic blood transfusion could potentially be an adverse prognostic indicator leading to increased post-operative cancer recurrence and reduced survival episodes (Ojima et al., 2009; Squires et al., 2015; Sun et al., 2015; Yang et al., 2015). In contrast, other studies revealed that peri-operative transfusion had limited influence on postoperative pulmonary infection and long-term mortality in patients with gastric cancer after gastrectomy (Rausei et al., 2013; Zhou et al., 2014; Xiao et al., 2014). Though accumulating evidence revealed the potential disadvantages of blood transfusion during gastrectomy in such cases of malignancy, the role of peri-operative blood transfusion in gastric cancer remained to be controversial.

Therefore, we conducted a retrospective study to survey the influence of blood transfusion on patients with gastric cancer receiving gastrectomy as well as the postoperative complications and mortality in Taiwan. 


\section{Materials and Methods}

From January 2011 to December 2014, adult patients (more than 20 years old) who were diagnosed as gastric cancer, including gastric adenocarcinoma, malignant gastrointestinal stromal tumor (GIST) and other gastric malignancies proven by histopathological verification, and subsequently received therapeutic resection at Far Eastern Memorial Hospital, were enrolled in the investigation. The clinical data were also requested via the electronic medical chart view, including patient age, gender, perioperative anemia states, usage of allogeneic transfusion of red blood cell (RBC) products, estimated blood loss (EBL) during operation, the length of hospital stay (LHS) from admission to discharge and operation to discharge, complication events post operation and the mortality rate at 6-month- and 1-year-interval. To evaluate peri-operative anemia states, the $\mathrm{Hb}$ level upon admission and before operation as well as the nadir $\mathrm{Hb}$ level post operation and the final $\mathrm{Hb}$ level before discharge were obtained. Postoperative infectious events with clinical symptoms and microbiological evidence, respiratory events (including lung edema, pleural effusion and respiratory failure), renal events (such as acute renal failure), cardiac events (such as acute myocardial infarction), bleeding events, thromboembolic events (including deep vein thrombosis, pulmonary embolism and cerebrovascular events) were considered as the complication events post operation.

Statistical analysis was performed using SPSS (version 19.0; SPSS Inc., Chicago, USA) statistical software. All data were expressed as mean \pm standard deviation (SD) or median (interquartile range; IQR). The unconditional logistic regression model was used for calculating the odds ratio (OR) in assessing the relationship of preoperative anemia, pre-, intra-, and post-operative RBC transfusion to the outcome (including the LHS from admission to discharge and operation to discharge, as well as complication events such as infectious events and acute renal failure), with the estimation of $95 \%$ confidence interval (CI). The Kaplan-Meier survival analysis was also performed with log-rank test. A P value less than 0.05 was considered statistically significant.

\section{Results}

A total of 116 patients being diagnosed as gastric cancer with histopathological verification and receiving curative gastrectomy were available at Far Eastern Memorial Hospital from 2011 to 2014, and the demographic characteristics of these patients were listed in Table 1. Among these, 98 were adenocarcinoma, 15 were malignant GIST and 3 belonged to other types of gastric malignancies (including 2 cases of gastric lymphoma and 1 case of neuroendocrine gastric carcinoma). Also, 60 were in early stage (stage I/II) and 56 were in later stage (stage III). The Hb level upon admission, pre-operative $\mathrm{Hb}$ level, post-operative nadir $\mathrm{Hb}$ level, and final $\mathrm{Hb}$ level before discharge were 11.2 $\pm 2.9,11.7 \pm 2.2,10.3 \pm 1.9$ and $11.1 \pm 1.7 \mathrm{~g} / \mathrm{dL}$, respectively. All the median units of $\mathrm{RBC}$ products transfused in pre-operative, peri-operative and post-operative periods with IQR were 0 with 0 to 2 units. The median EBL was $100 \mathrm{~mL}$ with IQR of 50 to $200 \mathrm{~mL}$. The LHS from admission to discharge and from operation to discharge were 16 and 13 days with IQR of 12 to 25 and 9 to 19 days, respectively. Additionally, the most common complication event post operation was the infectious event $(n=38,32.8 \%)$, followed by respiratory $(n=21,18.1 \%)$, renal $(n=13,11.2 \%)$ and bleeding events $(n=3,2.6 \%)$, respectively. The overall 6-month and 1-year mortality rates of patients with gastric cancer receiving gastrectomy were $3.4 \%$ and $6.9 \%$, respectively.

Univariate analysis for pre-operative anemia states and RBC transfusion in assessing the outcomes of patients with gastric cancer receiving gastrectomy were shown in Table 2. Pre-operative RBC transfusion seemed to be associated with post-operative infectious events (OR: 2.26, 95\% CI: 0.87-5.79, P=0.061) and acute renal failure (OR: $2.82,95 \% \mathrm{CI}$ : 0.70-10.78, $\mathrm{P}=0.078$ ), but with no statistical significance. Both pre-operative and intra- and post-operative transfusion of RBC products were markedly related to post-operative infectious events (OR: 3.70, 95\% CI: 1.43-9.58, P=0.002; OR: 8.20, 95\% CI: 3.11-22.62, $\mathrm{P}<0.001$, respectively). Additionally, intra- and post-operative transfusion of RBC products was significantly associated with prolonged LHS from admission to discharge (OR: 8.66, 95\% CI: 1.73-83.00, $\mathrm{P}=0.002)$ and post-operative acute renal failure (OR: 19.69, 95\% CI: 2.66-854.56, $\mathrm{P}<0.001)$.

Table 1. Demographic characteristics in patients with gastric cancer receiving gastrectomy

\begin{tabular}{|c|c|}
\hline Variables & \\
\hline Age (year) & $58(60-78)$ \\
\hline Gender (Male/Female) & $72 / 44$ \\
\hline \multicolumn{2}{|l|}{ Gastric cancer (n) } \\
\hline Adenocarcinoma & 98 \\
\hline Malignant GIST & 15 \\
\hline Other malignancies & 3 \\
\hline \multicolumn{2}{|l|}{ Stage $(n)$} \\
\hline $\mathrm{I} / \mathrm{II}$ & 60 \\
\hline III & 56 \\
\hline \multicolumn{2}{|l|}{ Hb level (g/dL) } \\
\hline Admission & $11.2 \pm 2.9$ \\
\hline Pre-OP & $11.7 \pm 2.2$ \\
\hline Nadir & $10.3 \pm 1.9$ \\
\hline Discharge & $11.1 \pm 1.7$ \\
\hline \multicolumn{2}{|l|}{ RBC product used (unit) } \\
\hline Pre-OP & $0(0-2)$ \\
\hline Peri-OP & $0(0-2)$ \\
\hline Post-OP & $0(0-2)$ \\
\hline $\operatorname{EBL}(\mathrm{mL})$ & $100(50-200)$ \\
\hline LHS from admission to discharge (day) & $16(12-25)$ \\
\hline LHS from OP to discharge (day) & $13(9-19)$ \\
\hline \multicolumn{2}{|l|}{ Complication events (n) } \\
\hline Infectious & 38 \\
\hline Respiratory & 21 \\
\hline Renal & 13 \\
\hline Bleeding & 3 \\
\hline \multicolumn{2}{|l|}{ Mortality rate $(\%)$} \\
\hline 6-month & 3.4 \\
\hline 1-year & 6.9 \\
\hline
\end{tabular}

Hb, hemoglobin; OP, operation; EBL, estimated blood loss; LHS, length of hospital stay. 
Table 2. Univariate Analysis of Peri-Operative Anemia States and RBC Transfusion in Predicting the Outcomes of Patients with Gastric Cancer Receiving Gastrectomy

\begin{tabular}{|c|c|c|c|c|}
\hline & \multicolumn{4}{|c|}{ LHS from admission to discharge (day) } \\
\hline & $\geq 30$ & $<30$ & Crude OR (95\% CI) & $\mathrm{P}$ value \\
\hline \multicolumn{5}{|c|}{ Pre-OP Hb level (g/dL) } \\
\hline$<10$ & 4 & 26 & $1.32(0.27-5.21)$ & 0.669 \\
\hline$\geq 10$ & 9 & 77 & & \\
\hline \multicolumn{5}{|l|}{ Pre-OP transfusion } \\
\hline Transfused & 5 & 26 & $1.85(0.43-7.07)$ & 0.312 \\
\hline Not transfused & 8 & 77 & & \\
\hline \multicolumn{5}{|c|}{ Intra-and post-OP transfusion } \\
\hline Transfused & 11 & 40 & $8.66(1.73-83.00)$ & 0.002 \\
\hline \multirow[t]{3}{*}{ Not transfused } & 2 & 63 & & \\
\hline & Infectious events & & & \\
\hline & With & Without & Crude OR (95\% CI) & $P$ value \\
\hline \multicolumn{5}{|c|}{ Pre-OP Hb level (g/dL) } \\
\hline$<10$ & 14 & 16 & $2.26(0.87-5.79)$ & 0.061 \\
\hline$\geq 10$ & 24 & 62 & & \\
\hline \multicolumn{5}{|c|}{ Pre-OP transfusion } \\
\hline Transfused & 17 & 14 & $3.70(1.43-9.58)$ & 0.002 \\
\hline Not transfused & 21 & 64 & & \\
\hline \multicolumn{5}{|c|}{ Intra-and post-OP transfusion } \\
\hline Transfused & 29 & 22 & $8.20(3.11-22.62)$ & $<0.001$ \\
\hline \multirow[t]{3}{*}{ Not transfused } & 9 & 56 & & \\
\hline & Acute renal failure & & & \\
\hline & With & Without & Crude OR (95\% CI) & $P$ value \\
\hline \multicolumn{5}{|c|}{ Pre-OP Hb level (g/dL) } \\
\hline$<10$ & 6 & 24 & $2.82(0.70-10.78)$ & 0.078 \\
\hline$\geq 10$ & 7 & 79 & & \\
\hline \multicolumn{5}{|l|}{ Pre-OP transfusion } \\
\hline Transfused & 3 & 28 & $0.80(0.13-3.44)$ & 0.754 \\
\hline Not transfused & 10 & 75 & & \\
\hline \multicolumn{5}{|c|}{ Intra-and post-OP transfusion } \\
\hline Transfused & 12 & 39 & $19.69(2.66-854.56)$ & $<0.001$ \\
\hline Not transfused & 1 & 64 & & \\
\hline
\end{tabular}

BC, red blood cell; Hb, hemoglobin; OP, operation; LHS, length of hospital stay; OR, odds ratio; CI, confidence interval.

(A)

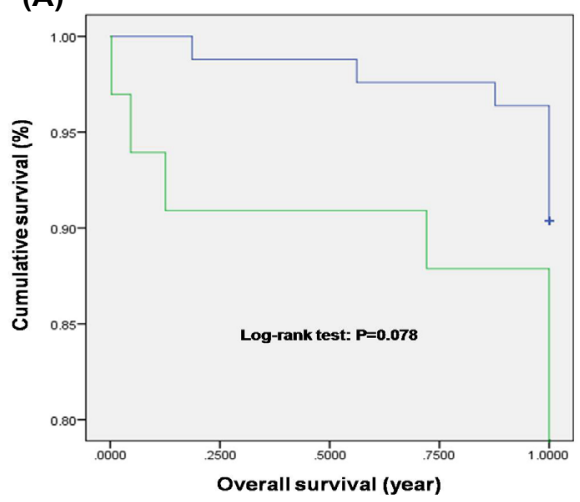

(B)

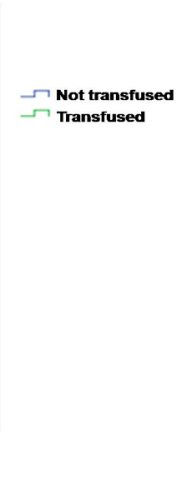

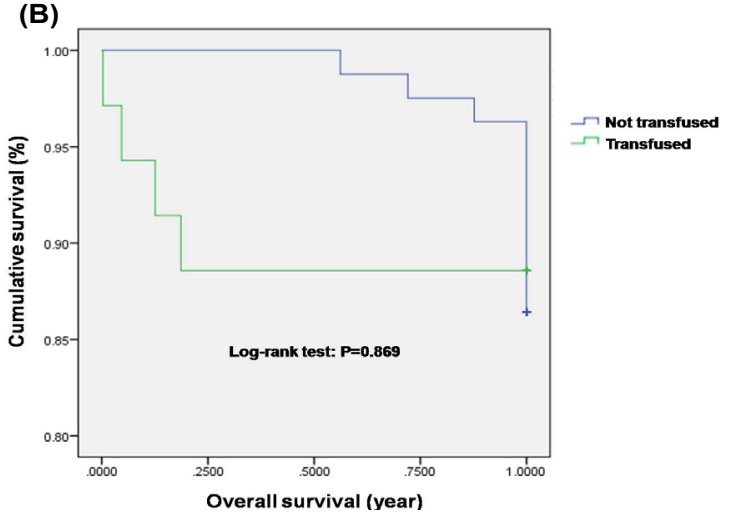

Figure 1. Kaplan-Meier survival analysis on patients with gastric cancer receiving gasrtectomy with and without intra-operative blood transfusion (A) as well as with and without post-operative blood transfusion (B)

Figure 1 revealed the overall survival rate of patients with gastric cancer receiving gastrectomy with 1-yearperiod follow-up. Intra-operative RBC transfusion seemed to be associated with higher mortality than those without in gastric cancer, but with no statistical significance $(\mathrm{P}=0.078$, Figure 1A). Besides, there was no remarkable difference between the groups with and without postoperative $\mathrm{RBC}$ transfusion $(\mathrm{P}=0.869$, Figure $1 \mathrm{~B})$.

\section{Discussion}

Our main finding revealed that RBC transfusion before, during and after surgery significantly increased the risk of post-operative infectious events in patients with gastric cancer receiving gastrectomy. Besides, intra- and postoperative RBC transfusion was remarkably associated with prolonged LHS from admission to discharge and 
post-operative acute renal failure. Furthermore, the risk of post-operative infectious events and acute renal failure was seemingly to be increased by RBC transfusion before gastrectomy, and the post-operative mortality was seemingly to be enhanced by RBC transfusion during gastrectomy.

It was highly prevalent that chronic anemia states were observed in patients with malignancies. The cancerrelated anemia was usually multi-factorial and could be contributed to chronic blood loss, malnutrition, immune response, as well as metabolite homeostasis (Macciò et al., 2015). According to literature review, pre-operative anemia states in malignancies were associated with increased morbidity and mortality (Yu et al., 2011; Gierth et al., 2015). Theoretically, it was essential that pre-operative rectification of anemic condition in patients who would undergo surgical management. However, it had been controversial that whether allogeneic blood transfusion before, during and after surgery affected disease progression and patient outcomes. Indeed, accumulating evidence proved that excess blood transfusion during surgery might not improve but even aggravated the prognosis and increased post-operative complications, especially in curative surgery for malignancies (Meng et al., 2013; Aquina et al., 2015; Diaz-Cambronero et al., 2015; Li et al., 2015; Prescott et al., 2015). Hence, oncologic surgeries without blood transfusion were developed without increased post-operative complications by means of replacement fluid administration, autologous blood infusion, parenteral iron supplement and the use of erythropoietic-stimulating agents (Lee et al., 2014; Borstlap et al., 2015; Diaz-Cambronero et al., 2015; Inoue et al., 2015). Recently, it was reported that such novel methods for blood preservation could be practical in oncologic surgeries, particularly for the selected populations such as patients with uncommon blood types or multiple RBC allo-antibodies, as well as Jehovah's Witnesses. There was, nevertheless, no sufficient evidence to prove that some of these blood preservation methods could benefit the outcomes of patients with malignancies (Devon et al., 2009; Kang et al., 2012). Further research was required in evaluating the safety of transfusionfree cancer management as well as the efficacy of other transfusion alternatives (Oh et al., 2010).

The main limitations of our study included the limited case number with the retrospective study design. Besides, the monitor interval could be inadequate for the observation of disease evolution such as cancer recurrence or metastasis, complication events and long-term mortality post operation, suggesting bias to a certain extent. Multicenter prospective research for assessing the effect of blood transfusion during curative surgery on the prognosis of gastric cancer might be considered.

In conclusion, our study indicated that pre-, peri- and post-operative RBC transfusion significantly increased the risk of infection events post surgery in patients with gastric cancer receiving gastrectomy. Additionally, peri- and post-operative RBC transfusion significantly prolonged LHS from admission to discharge and increased the risk of post-operative infectious events and acute renal failure. Also, the overall survival was seemingly to be decreased by peri-operative RBC transfusion in gastric cancer. Our survey indicated that peri-operative RBC transfusion could have an adverse effect on patients who had gastric cancer and received gastrectomy. Hence, unnecessary blood transfusion before, during and after curative gastrectomy should be avoided in gastric cancer.

\section{Acknowledgements}

We thankfully acknowledge the financial support from Far Eastern Memorial Hospital (FEMH-2016-D-052)

\section{References}

Aquina CT, Blumberg N, Probst CP, et al (2015). Significant Variation in Blood Transfusion Practice Persists following Upper GI Cancer Resection. J Gastrointest Surg, 19, 192737.

Borstlap WA, Buskens CJ, Tytgat KM, et al (2015). Multicentre randomized controlled trial comparing ferric(III) carboxymaltose infusion with oral iron supplementation in the treatment of preoperative anaemia in colorectal cancer patients. BMC Surg, 15, 78.

Devon KM, McLeod RS (2009). Pre and peri-operative erythropoietin for reducing allogeneic blood transfusions in colorectal cancer surgery. Cochrane Database Syst Rev, $\mathbf{1}, 7148$.

Diaz-Cambronero O, Matoses-Jaén S, Garcia-Claudio N, et al (2015). Preoperative management of anemia in oncologic surgery. Rev Esp Anestesiol Reanim, 62, 45-51.

Gantt CL (1981). Red blood cells for cancer patients. Lancet, 12, 363 .

Gierth M, Mayr R, Aziz A, et al (2015). Preoperative anemia is associated with adverse outcome in patients with urothelial carcinoma of the bladder following radical cystectomy. $J$ Cancer Res Clin Oncol, 141, 1819-26.

Inoue S, Miyoshi T, Aoyama M, et al (2015). A case of thoracic esophageal cancer undergone esophagectomy after induction chemotherapy in a Jehovah's Witness. J Med Invest, 62, 264-7.

Kang RY, Lee J, Lee YH, et al (2012). Impact of erythropoiesisstimulating agents on red blood cell transfusion in Korea. Int J Clin Pharm, 34, 651-7.

Lee BW, Park MG, Cho DY, et al (2014). Preoperative erythropoietin administration in patients with prostate cancer undergoing radical prostatectomy without transfusion. Korean J Urol, 55, 102-5.

Li XX, Meng J, Sun GP, et al (2015). Effects of perioperative blood transfusion on the prognosis in hereditary and sporadic colon cancer. Biomarkers, 20, 481-6.

Macciò A, Madeddu C, Gramignano G, et al (2015). The role of inflammation, iron, and nutritional status in cancer-related anemia: results of a large, prospective, observational study. Haematologica, 100, 124-32.

Meng J, Lu XB, Tang YX, et al (2013). Effects of allogeneic blood transfusion in patients with stage II colon cancer. Asian Pac J Cancer Prev, 14, 347-50.

Oh SY, Kim SH, Kwon HC, et al (2010). Bloodless cancer treatment results of patients who do not want blood transfusion: single center experience of 77 cases. Support Care Cancer, 18, 1341-6.

Ojima T, Iwahashi M, Nakamori M, et al (2009). Association of allogeneic blood transfusions and long-term survival of patients with gastric cancer after curative gastrectomy. $J$ Gastrointest Surg, 13, 1821-30.

Prescott LS, Aloia TA, Brown AJ, et al (2015). Perioperative 
blood transfusion in gynecologic oncology surgery: analysis of the national surgical quality improvement program database. Gynecol Oncol, 136, 65-70.

Rausei S, Ruspi L, Galli F, et al (2013). Peri-operative blood transfusion in gastric cancer surgery: prognostic or confounding factor? Int J Surg, 11, S100-3.

Squires MH 3rd, Kooby DA, Poultsides GA, et al (2015). Effect of perioperative transfusion on recurrence and survival after gastric cancer resection: a 7-institution analysis of 765 patients from the us gastric cancer collaborative. J Am Coll Surg, 221, 767-77.

Sun C, Wang Y, Yao HS, et al (2015). Allogeneic blood transfusion and the prognosis of gastric cancer patients: systematic review and meta-analysis. Int J Surg, 13, 102-10.

Tsai HW, Hsieh FC, Chang CC, et al (2015). Clinical practice of blood transfusion in orthotopic organ transplantation: a single institution experience. Asian Pac J Cancer Prev, 16, 8009-13.

Xiao H, Zuo C, Ouyang Y, et al (2014). Multivariate analysis of risk factors for pulmonary infection after radical gastrectomy for gastric cancer. Zhonghua Yi Xue Za Zhi, 94, 3310-3.

Yang K, Chen XZ, Zhang WH, et al (2015). Effect of Perioperative Transfusion on Survival and Morbidity for Gastric Cancer Patients with Gastrectomy. J Am Coll Surg, 221, 995-6.

Ye X, Liu J, Chen Y, et al (2015). The impact of hemoglobin level and transfusion on the outcomes of chemotherapy in gastric cancer patients. Int J Clin Exp Med, 8, 4228-35.

Yu JC, Ge JN, Tang Y, et al (2011). Multicenter cross-sectional study of anemia in patients with gastric and colorectal cancer before and after the operation. Zhonghua Wai Ke Za Zhi, 49, 53-6.

Zhou HY, Yi W, Wang J, et al (2014). Association of perioperative allogeneic blood transfusions and prognosis of patients with gastric cancer after curative gastrectomy. Am J Surg, 208, 80-7. 